\title{
Factors Determining Discharge Location in EDM
}

\author{
Masanori Kunieda and Takanobu Nakashima \\ (Received April 30, 1997)
}

\author{
Department of Mechanical Systems Engineering \\ Tokyo University of Agriculture \& Technology \\ Tokyo 184, Japan
}

\begin{abstract}
We describe the factors which affect the determination of the discharge location in electrical discharge machining. The main factors investigated are the working gap width, the debris particle concentration, the surface area, and the degree of plasma deionization. It is found that, even if there are no debris particles drifting in the working gap, discharge does not always occur at the point where the gap is narrowest due to the effect of the surface area. It is also found that the preceding discharge does not affect the location of the subsequent discharge as long as the plasma formed by the preceding discharge is deionized during the discharge interval. In a series of pulse discharges, since numerous chains of debris particles grow over the working surface, the influence of the debris particle concentration becomes more dominant than that of the gap width distribution, which can lead to process instability.
\end{abstract}

Key words: EDM, discharge location, gap width, debris particle, plasma

\section{INTRODUCTION}

For a stable continuation of pulse discharges in electrical discharge machining (EDM), it is essential that the discharge locations are uniformly distributed over the working surface. If the successively occurring pulse discharges are concentrated at one location, both the number density of debris particles and the surface temperature on the electrodes are increased, and as a result, the dielectric breakdown strength of the gap will be weakened. This will cause an enhanced concentration of discharge at the same location, which will result in not only deteriorated machining accuracy due to the uneven distribution of the gap width but also machining instability.

Therefore, we must understand the mechanism of how the discharge location is determined. So far it has been believed that a discharge occurs at a point where the gap is shortest. If this is true, when a debris particle with a diameter of $5 \mu \mathrm{m}$ is placed in a gap with a width of $20 \mu \mathrm{m}$, electric breakdown will occur at the point where the debris particle is placed. In this experiment, the gap is filled with a clean EDM working oil and the surface roughness of the electrodes is $0.8 \mu \mathrm{m}$ Rmax. However, the experimental results show that in most cases, the discharge crater cannot be found at the point where the debris particle was placed. In place of inserting a debris particle, we form an indentation using a punch. The ridge height of the indentation is almost the same as the diameter of the debris particle. Even in this situation, it is rare that the discharge breaks at the ridge. Hence it is found that a discharge does not always occur at a point where the gap is shortest. This is the reason why we aim to elucidate the dominant factors which affect the determination of the next discharge location in EDM process.

\section{INFLUENCE OF GAP WIDTH}

First of all we investigated the influence of the gap width on the probability of occurrence of discharge when the discharge gap is filled with a clean dielectric liquid. As shown in Fig. 1, a flat anode faces two flat cathodes $\mathrm{A}$ and $\mathrm{B}$ both with surface areas of $10 \mathrm{~mm} \times 10 \mathrm{~mm}$. Cathode $A$ is higher than cathode $\mathrm{B}$ by $s$, and the gap width between cathode A and the anode is $g$. The electrode material is mild steel (SS41) and the open voltage applied between the anode and cathode is $260 \mathrm{~V}$. Figure 2 shows the change in the probability of occurrence of discharge on cathode $\mathrm{A}$ as $g$ and $s$ are changed. All the surfaces of the electrodes are 


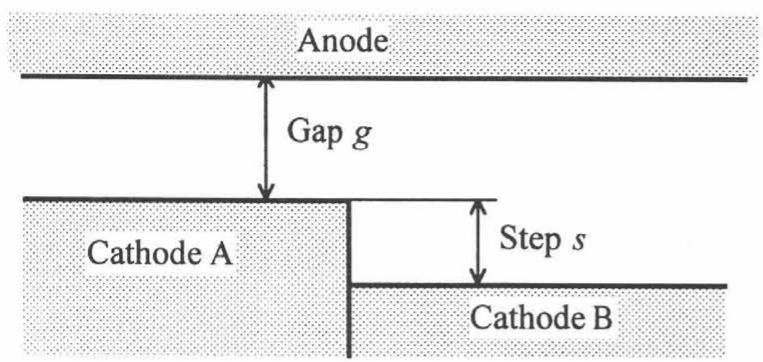

Fig. 1 Setup for examining the influence of the gap width on the probability of discharge occurrence

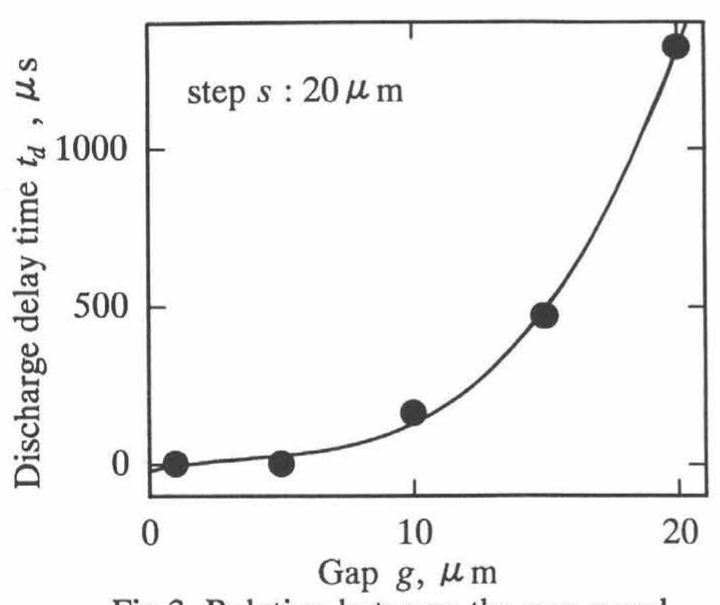

Fig.3 Relation between the gap $g$ and the discharge delay time

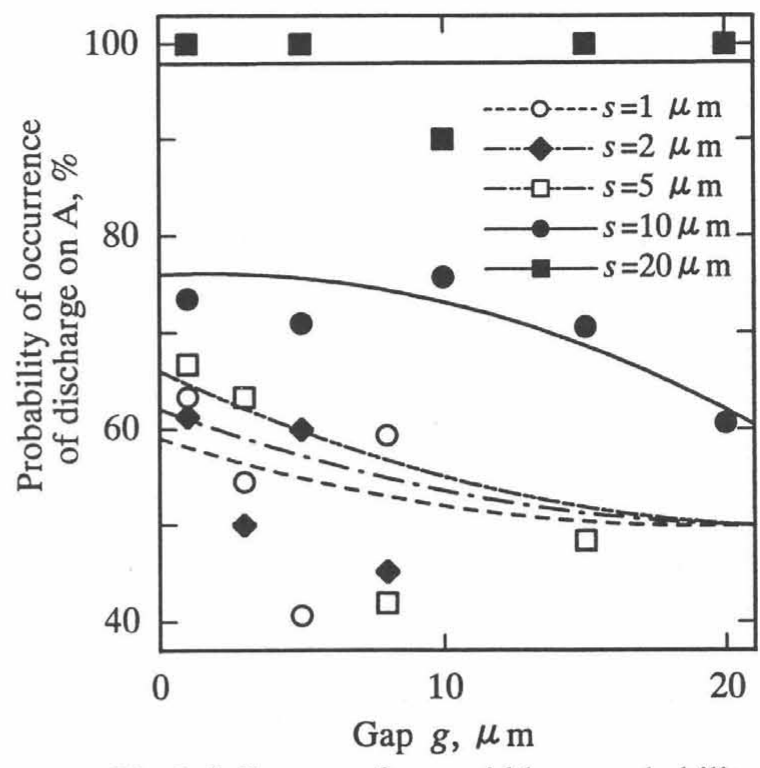

Fig.2 Influence of gap width on probability of discharge occurrence

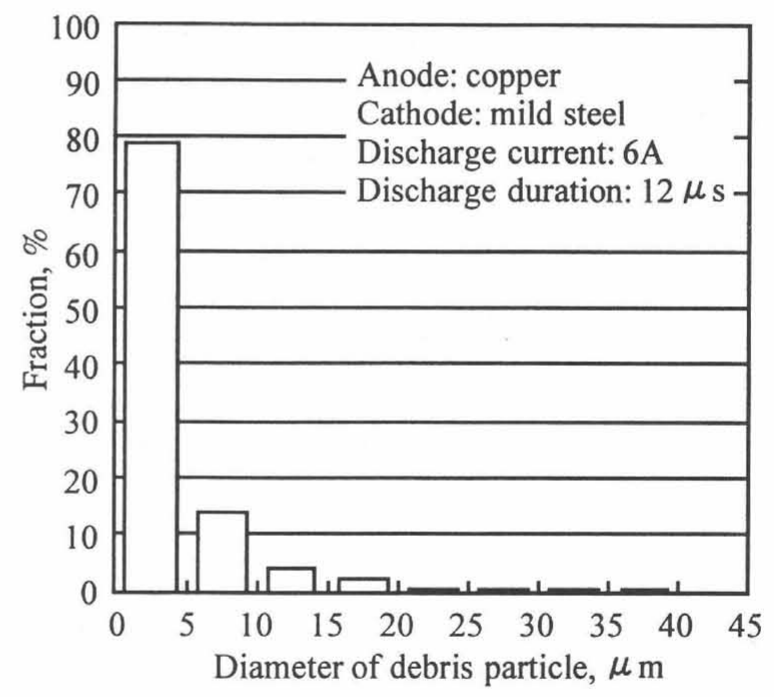

Fig,4 Distribution of diameters of debris particles

obtained under such conditions are not included in the statistics.

The overall results show that when $g$ is constant the probability increases with the increase in $s$. When $s$ is constant the probability increases with the decrease in $g$ and converges to $50 \%$ on increasing $g$. For cathode A, since the gap is small, discharge always occurs on the cathode A side when $s=20 \mu \mathrm{m}$; however, when $s$ is smaller than (or equal to) $10 \mu \mathrm{m}$, 


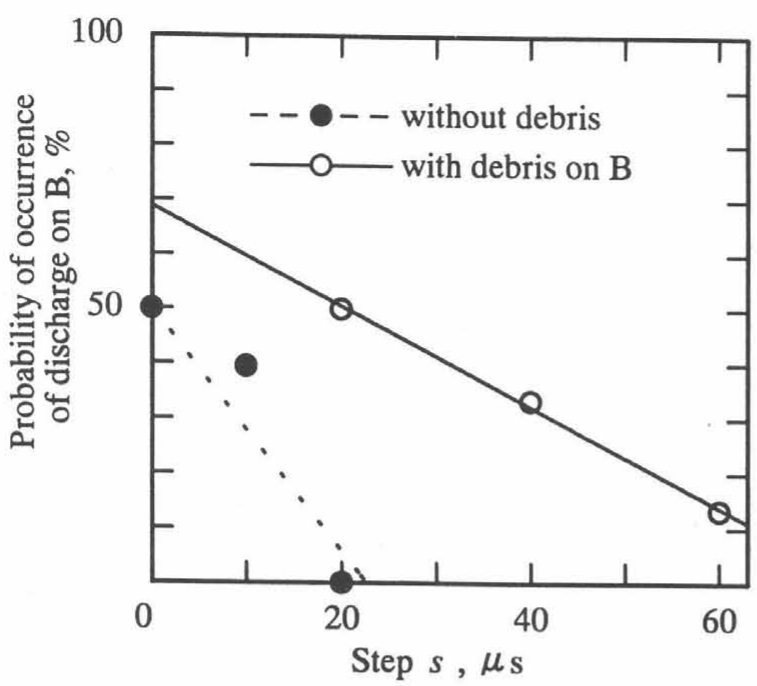

Fig.5 Influence of presence of debris particles on probability of discharge occurrence

the probability of occurrence of discharge is not $100 \%$ even if the gap on the cathode A side is small.

Figure 3 shows the relationship between $g$ and the discharge delay time when $s$ is $20 \mu \mathrm{m}$ and the discharge occurred on cathode A. The discharge delay time increases exponentially with increasing $g$.

\section{INFLUENCE OF DEBRIS PARTICLES}

Next we examined the influence of the presence of debris particles in the gap on the probability of discharge, using the same setup as shown in Fig. 1. To introduce the debris particles onto cathode $\mathrm{B}$, the surface of cathode B was machined by EDM using another flat copper anode with the pulse conditions of discharge current of $6 \mathrm{~A}$ and discharge duration of $12 \mu \mathrm{s}$. After machining for 50 seconds, the copper anode was moved away from the machining area carefully and gently in order to maintain all debris particles on the surface of cathode B. The distribution of the diameters of debris particles produced under the above pulse conditions was measured using an optical microscope and is shown in Fig. 4. The surface roughness of the machined cathode B was $10 \mu \mathrm{m} \mathrm{Rmax}$ and the diameter of most of the debris particles was less than $5 \mu \mathrm{m}$.

The measured probability of occurrence of discharge on cathode B is shown in Fig. 5. Here, $g$ is constant at $20 \mu \mathrm{m}$. Since the diameter of most of

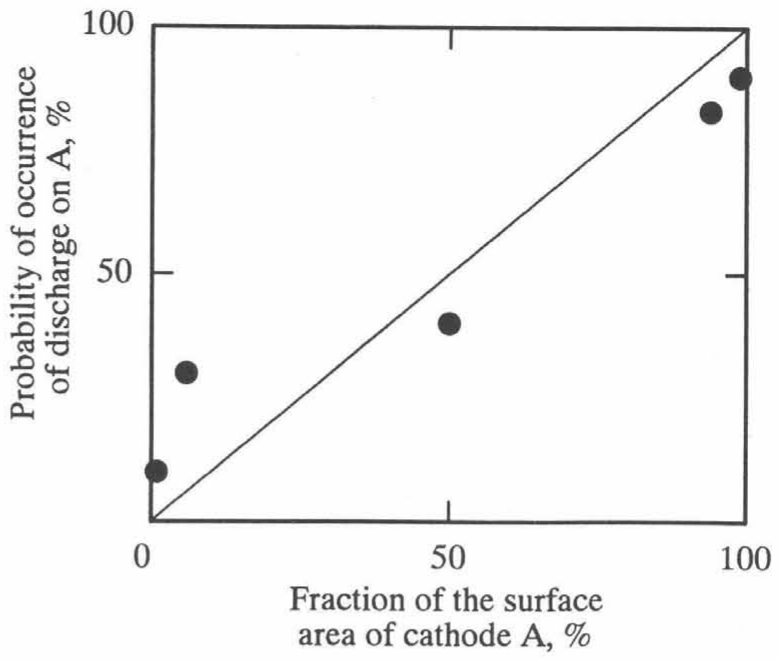

Fig. 6 Influence of the surface area of the electrode on the probability of discharge occurrence

the debris particles is less than $5 \mu \mathrm{m}$, if individual debris particles are deposited on the surface without being linked in series to form chains, the actual gap on cathode B, which is the sum of $g$ and $s$ subtracted by the diameter, is still much larger than $g$. When $s$ is substantially large, for example, $60 \mu \mathrm{m}$, the diameter of $5 \mu \mathrm{m}$ is negligible compared to $s$. Nevertheless, the probability of occurrence of discharge on cathode B is not zero even when $s=60 \mu \mathrm{m}$, while, without a debris particle, the probability is zero when $s$ is larger than $20 \mu \mathrm{m}$.

This result can be explained in terms of the movement of debris particles in the gap, which was observed by Suda and Sata ${ }^{1)}$ and analyzed by Kunieda and Mori $^{2)}$ and Kunieda and Yanatori ${ }^{3)}$. They found that the debris particles move between the anode and cathode in a direction perpendicular to the electrode surfaces due to electrophoresis. They also observed that some particles are linked in series to form chains parallel to the electric field. Therefore, it is considered that, in the gap, there are numerous chains of particles which almost bridge the gap and that discharge occurs where the end of a chain is closest to the opposite electrode surface.

\section{INFLUENCE OF SURFACE AREA OF ELECTRODE}

In section 2 it was clarified that the probability of 


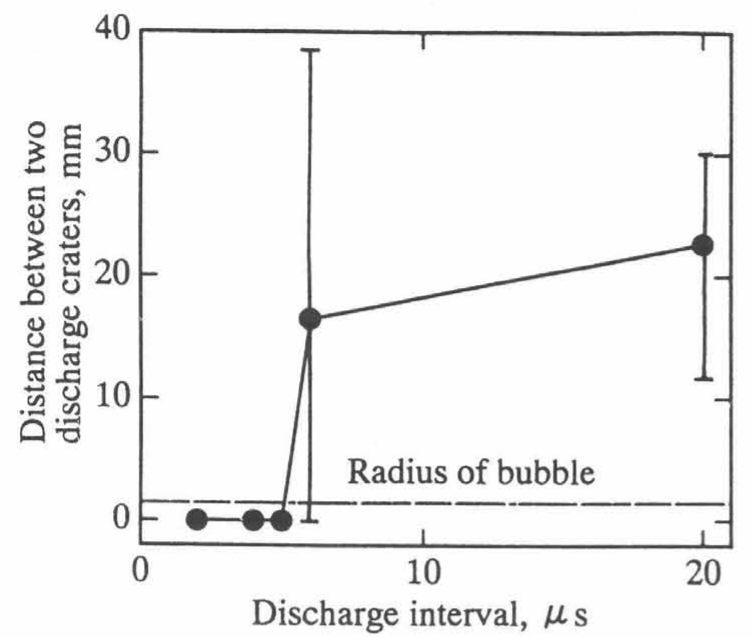

Fig. 7 Influence of the plasma formed by the first discharge on the discharge location of the second discharge

occurrence of discharge on cathode $\mathrm{B}$, for which the gap is wider than that for cathode $\mathrm{A}$, is not zero. This suggests that the probability of occurrence of discharge on a surface is proportional to its surface area. Hence we here examine the influence of the ratio of the surface areas of cathodes $\mathrm{A}$ and $\mathrm{B}$ on the probability of occurrence of discharge on cathode A.

Figure 6 shows the relationship between the fraction of the surface area of cathode $A$ to the whole working surface area and the probability of occurrence of discharge on cathode A when $g$ and $s$ are $10 \mu \mathrm{m}$ and $3 \mu \mathrm{m}$, respectively. For each fraction of the surface area, the probability was obtained from 30 trials of single discharge as described in Section 2.

Although the gap on cathode $\mathrm{A}$ is narrower than that on cathode $\mathrm{B}$, the probability is almost proportional to the fraction of the surface area of cathode A. This explains the experimental result mentioned in the introduction that, in most cases, the discharge crater cannot be found at the point where only one debris particle was placed between plane parallel electrodes. This is because the projected area of the debris particle in the direction normal to the electrode surface is negligible compared with the total area of the electrodes $(100 \mathrm{~mm} \times 100 \mathrm{~mm})$.

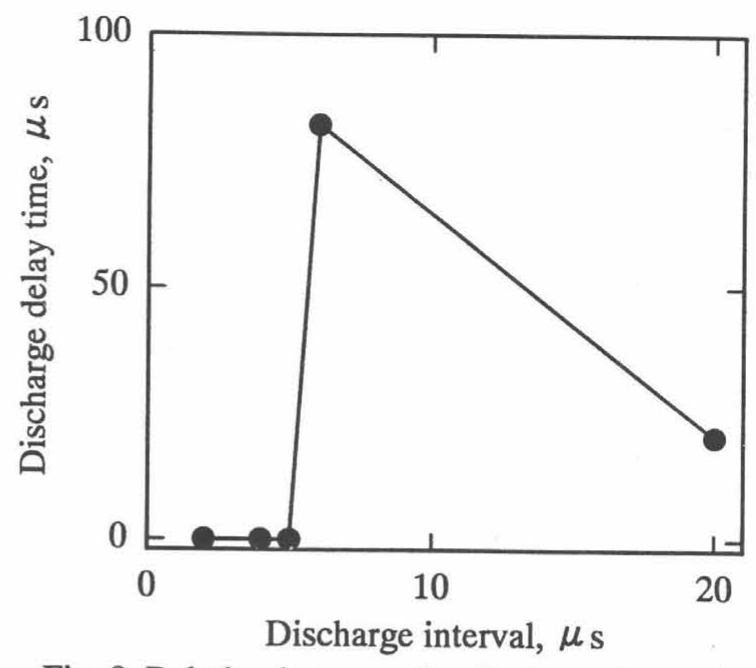

Fig. 8 Relation between the discharge interval and the discharge delay time for the second discharge

\section{INFLUENCE OF PLASMA EXTINCTION}

The influence of the plasma formed by the first pulse discharge between parallel plane electrodes on the discharge location of the second pulse discharge is investigated. Both the anode and cathode used in the experiment are mild steel (SS41) and have the same surface area of $50 \mathrm{~mm} \times 50 \mathrm{~mm}$. The two surfaces are ground to a surface roughness of 0.10 to $0.16 \mu \mathrm{m} \mathrm{Rmax}$, and two pulse discharges are induced successively with a certain inter-pulse interval. After the discharges, the distance between the two discharge craters formed on one of the electrodes is measured. The gap between the electrodes is $10 \mu \mathrm{m}$ and filled with a clean EDM working oil. Table 1 shows the pulse conditions adopted.

Figure 7 shows the influence of the discharge interval between two consecutive pulse discharges on the distance between two discharge craters. The discharge delay time for the second discharge was also measured. The average discharge delay time for each discharge interval is shown in Fig. 8. For each discharge interval, 20 trials were carried out.

When the discharge interval is less than or equal to $5 \mu \mathrm{s}$, the second discharge occurs consistently on the discharge crater formed by the first discharge, and the discharge delay time is almost zero. However, when the discharge interval is longer than 
Table 1 Pulse conditions used in the experiment to examine the influence of the plasma formed by the first discharge on the discharge location of the second discharge.

\begin{tabular}{|l|c|}
\hline Discharge duration, $\mu_{\mathbf{s}}$ & 120 \\
\hline Discharge interval, $\mu_{\mathbf{s}}$ & $2,4,5,6,20$ \\
\hline Discharge current, A & 9 \\
\hline Open voltage, $\mathrm{V}$ & 260 \\
\hline
\end{tabular}

or equal to $6 \mu \mathrm{s}$, the positions are scattered as if there is no relationship between the first and the second discharge, and the discharge delay time is not zero. Therefore, when the discharge interval is less than $6 \mu \mathrm{s}$, the plasma generated by the first discharge cannot be deionized and the dielectric breakdown strength of the gap cannot be recovered by the time the open voltage for the second discharge is applied.

This conclusion is consistent with the calculation result obtained by Hayakawa et al. ${ }^{4)}$ who estimated the time required for the plasma to be extinguished to be 1 to $2 \mu \mathrm{s}$. They also verified their calculation result by measuring the minimum discharge interval necessary for a finite discharge delay time to be observed before the second discharge. The minimum discharge delay time obtained by them was $3 \mu \mathrm{s}$.

The critical pulse interval was also addressed by Snoeys et al. ${ }^{5)}$ who found that the danger for arcing increases when the pulse interval is less than around $3-4 \mu \mathrm{s}$ from the measurement of frequency of occurrence of various pulse types when machining goes.

The chain line in Fig. 7 indicates the average measured radius of the bubble generated by each pulse discharge. From the observation of the distribution of debris particles which are generated and scattered around the discharge spot after a single pulse discharge between plane parallel electrodes,
Yoshida and Kunieda ${ }^{\text {) }}$ found that the debris particles are distributed on the periphery of the disklike bubble. Nevertheless, the second discharge occurs at a point unrelated to the size of the bubble even though there is no debris particle at the point. Neither does the ridge of the crater formed by the first discharge affect the discharge location of the second discharge, which is consistent with the result of the preliminary experiment described in the introduction.

Even for two consecutive pulse discharges, the discharge location for the second discharge is not related to the discharge location for the first discharge when the discharge interval is long enough. Hence in a series of pulse discharges, it is much more likely that the preceding discharge does not affect the succeeding discharges, because numerous chains of debris particles grow over the working surface. In fact, Kunieda and Kojima ${ }^{7}$ measured the distribution of discharge locations in a series of successive pulse discharges and found that under a stable machining state the distribution of discharge locations is random over the working surface.

\section{DISCUSSIONS}

Based on the results described above, the cause of machining instability in EDM is discussed. As Kunieda and Kojima $^{7}$ discussed based on the measurement of the discharge locations, when the working area is deepened, the flow of the working liquid becomes difficult and the working gap becomes contaminated with debris particles. The uneven distribution of debris particles causes localization of discharge spots, which results in a further increase in the debris particle concentration. In this way, even if the gap in the high-debrisconcentration area becomes wider than that in other working areas, the discharge is localized to the same area because the debris particles are linked in series to form numerous chains of particles. However, if the step, which is defined as the difference in the gap between the high and the low debris concentration areas, becomes large enough, the discharge location moves to another area. Repetition of the above cycle results in the periodical movement of the area of discharge localization, which was observed by Kunieda and Kojima ${ }^{7}$.

It is known that the plasma channel is cooled primarily due to the heat conduction into the anode 
and cathode and that the heat transfer through convection or radiation can be neglected ${ }^{4}$. Therefore, when the degree of localization of discharge spots increases markedly, the surface temperature of the electrodes in the area of discharge localization increases followed by a reduction of the heat conduction from the plasma to the electrodes. Hence, deionization of the plasma channel during the discharge interval becomes difficult, even if the discharge interval is set longer than the necessary minimum. This results in an abnormal discharge leading to process instability.

\section{CONCLUSIONS}

The mechanism of determining the discharge location was investigated and the cause of the instability of the EDM process was discussed. The main factors on which we focused are the working gap width, the debris particle concentration, the surface area, and the degree of plasma deionization. The following conclusions were obtained:

(1) When the dielectric fluid is not contaminated, the probability of discharge in the area where the gap is narrow is high. In terms of the surface area, however, the discharge does not always occur at the point where the gap is narrowest.

(2) For two consecutive pulse discharges, the first discharge does not affect the discharge location of the second one as long as the plasma formed by the first discharge is deionized during the discharge interval. The minimum discharge interval necessary for the plasma to be deionized is around $6 \mu \mathrm{s}$ under the pulse conditions used in this study.

(3) The debris particles generated and distributed around the first discharge crater in a clean dielectric fluid do not affect the next discharge location because of the effect of the surface area. However, in a series of pulse discharges in which numerous chains of debris particles grow over the working surface, the influence of the debris particle concentration becomes more dominant than that of the gap width distribution. This seems to be the main reason for the occurrence of the discharge localization.

\section{REFERENCES}

1) T. Suda, T. Sata: Movement of Electrically Conductive Particles in the Gap of EDM Process, J. of JSEME, 7, 14, (1974), pp.19-28 (in Japanese).

2) M. Kunieda, M. Mori: Relation between Debris Concentration and Discharge Gap Length in EDM Process, Proc. of Asian Electrical-machining Symposium '95, pp.1-6.

3) M. Kunieda, K. Yanatori: Study on Debris Movement in EDM Gap, IJEM, 2, (1997), pp.4349.

4) S. Hayakawa, H. Xia, M. Kunieda and N. Nishiwaki: Analysis of Time Required to Deionize an EDM Gap during Pulse Interval, ICHMT Symposium on Molecular and Microscale Heat Transfer in Materials Processing and Other Applications, Vol.1, (1996), pp.368-377.

5) R. Snoeys, D. Dauw and J. P. Kruth: Selection of Optimal Working Conditions in EDM, Proceedings of 20th MTDR Conf., (1979), pp.583-590.

6) M. Yoshida and M. Kunieda: Study on the Distribution of Scattered Debris Generated by a Single Pulse Discharge in EDM Process, JSEME, 30, 64, (1996), 27-36 (in Japanese).

7) M. Kunieda, H. Kojima: On-line Detection of EDM Spark Locations by Multiple Connection of Branched Electric Wires, Annals of the CIRP, 39, 1, (1990), pp.171-174. 